Acta Zoológica Mexicana (nueva serie), Volumen 37, 1-9.

https://doi.org/10.21829/azm.2021.3712309

Artículo original

\title{
Brúquidos (Insecta: Coleoptera) asociados a semillas de fabáceas en el Norte de Sinaloa, México
}

\section{Bruchids (Insecta: Coleoptera) associated with fabaceae seeds in North Sinaloa, Mexico}

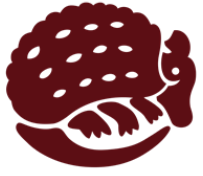

OPEN ACCESS

*Autor corresponsal:

Desús Alberto Acuña-Soto coleoptero77@hotmail.com

Cita:

López-Mora, J. F., Isiordia-Aquino,

N., Lugo-García, G. A., Flores-

Canales, R. J., Reyes-Olivas, A., Acuña-Soto, J. A. (2021) Brúquidos (Insecta: Coleoptera) asociados a semillas de fabáceas en el Norte de

Sinaloa, México. Acta Zoológica

Mexicana (nueva serie), 37, 1-9.

10.21829/azm.2021.3712309

elocation-id: e3712309

Recibido: 09 septiembre 2020 Aceptado: 29 diciembre 2020 Publicado: 05 febrero 2021

\author{
${ }^{1}$ Jesús Fernando LÓPEZ-MORA, (iD) ${ }^{2}$ NéSTOR ISIORDIA-AQUINO, \\ (iD) ${ }^{3}$ GABRIEL ANTONIO LUGO-GARCÍA, (iD) 2 'rICARDO JAVIER FLORES- \\ CANALES, (iD ${ }^{3}$ Álvaro REYES-OLIVAS, (iD 4*Jesús Alberto ACUÑA- \\ SOTO
}

\footnotetext{
${ }^{1}$ Estudiante de Posgrado CBAP. Universidad Autónoma de Nayarit. Km 9 carretera Tepic-Puerto Vallarta, Colonia Centro, Xalisco, Nayarit, México. C.P. 63780.

Unidad Académica de Agricultura. Universidad Autónoma de Nayarit. Km 9 carretera Tepic-Puerto Vallarta, Colonia Centro, Xalisco, Nayarit, México. C.P. 63780.

${ }^{3}$ Universidad Autónoma de Sinaloa, Colegio de Ciencias Agropecuarias, Facultad de Agricultura del Valle del Fuerte, Calle 16 y Avenida Japaraqui, C.P. 81110. Juan José Ríos, Ahome, Sinaloa, México.

${ }^{4}$ Instituto Tecnológico Superior de Tlatlaquitepec. Km $122+600$, Carretera Federal Amozoc - Nautla, C.P. 73907. Almoloni, Tlatlaquitepec, Puebla, México.
}

Editor responsable: Arturo Bonet Ceballos

RESUMEN. Las fabáceas representan una importante fuente de recursos al ser utilizadas en la producción de carbón, alimentación del ganado y personas, además de evitar erosiones del suelo; estas plantas se encuentran amenazadas en su producción por la presencia de brúquidos o gorgojos (Insecta: Coleoptera), quienes se alimentan de sus semillas para sobrevivir, causando pérdidas económicas en la región. En 2017 se realizaron recolectas de semillas en cuatro municipios del norte de Sinaloa, con el objetivo de identificar las especies de brúquidos asociados a fabaceas y evaluar sus daños sobre la germinación de semillas. Se identificaron 25 especies de fabáceas: Ahome (7), El Fuerte (6), Guasave (6) y Choix (6). Del total de 68,340 semillas, emergieron 19,396 gorgojos adultos, agrupados en siete géneros y 14 especies, con dominancias en 
las especies de Mimosestes (3), Acanthoscelides (3), Merobruchus (3) y Stator (2). De las especies vegetales, 17 fueron atacadas por una sola especie de brúquido, y seis por dos; en Ahome la especie plaga más perjudicial fue Merobruchus santarosae, con $43.9 \%$ y $33.1 \%$ de daños a Mariosouza coultieri y M. acatlensis, respectivamente; en El Fuerte, Acanthoscelides desmanthi causó daños del $38.95 \%$ a Sesvania herbaceae y del $29.48 \%$ a S. occidentalis; en Guasave, los mayores daños correspondieron a Callosobruchus maculatus sobre Cicer arietinum (41.3\%), Mimosestes ulkei sobre Parkinsonia aculeata (40.6\%) y M. mimosae sobre $P$. florida (37.7\%); en Choix los mayores daños correspondieron a Merobruchus insolitus sobre Albizia sinaloensis (34.7\%) y Amblycerus acapulcencis sobre Caesalpinia cacalaco (29.1\%).

Palabras clave: Bruchidae; Fabaceae; gorgojos; daños a semillas

ABSTRACT. Fabaceae represent an important source of resources when are used in coal production, feeding of livestock and people, in addition to avoiding soil erosions; these plants are threatened in their production by the presence of bruchids or weevils (Insecta: Coleoptera), who feed on their seeds to survive, causing economic losses in the region. In 2017, seed collections were carried out in four municipalities in the North of Sinaloa, with the objective of identifying the bruquid species associated with Fabaceae and assessing their damage on the germination of seeds. 25 species of Fabaceae were identified: Ahome (7), El Fuerte (6), Guasave (6) and Choix (6). From 68,340 seeds, 19,396 adult weevils emerged, grouped into seven genera and 14 species, with dominance in species of Mimosestes (3), Acanthoscelides (3), Merobruchus (3), and Stator (2). Of the plant species, 17 were attacked by a kind of bruquid and six by two; in Ahome the most harmful pest species was Merobruchus santarosae, with $43.9 \%$ and $33.1 \%$ damage to Mariosouza coultieri and M. acatlensis, respectively; in El Fuerte, Acanthoscelides desmanthi caused $38.95 \%$ damage to Sesvania herbaceae and $29.48 \%$ to S. occidentalis; in Guasave, the greatest damages corresponded to Callosobruchus maculatus on Cicer arietinum (41.3\%), Mimosestes ulkei on Parkinsonia aculeata (40.6\%) and M. mimosae on P. florida (37.7\%); in Choix, the greatest damages corresponded to Merobruchus insolitus on Albizia sinaloensis (34.7\%) and Amblycerus acapulcencis on Caesalpinia cacalaco (29.1\%).

Key words: Bruchidae; Fabaceae; weevils; seeds damage

\section{INTRODUCCIÓN}

Las especies vegetales pertenecientes a la familia Fabaceae (Leguminosae) son utilizados como recursos maderables, en la producción de carbón y alimentación para el ganado, e incluso para las personas, como en el caso de mezquite (Prosopis juliflora (Sw.) DC. 1825). Estos árboles son resistentes a las sequías y evitan la erosión del suelo (Aguado \& Suárez, 2006). Esta familia es de distribución cosmopolita, con alrededor de 730 géneros y 19,400 especies, que incluyen hierbas perennes, árboles y arbustos (Harden, 2002).

De las casi 280,000 especies de plantas con flores a nivel mundial, las Fabaceae son uno de los grupos más abundantes, junto con las orquídeas (Orquideaceae) y las compuestas (Asteraceae); de hecho, en México, esta familia es la segunda en cuanto a número de especies 
$(1,724)$ y de éstas, el $51.9 \%$ son endémicas del país (Sousa \& Delgado, 1993); aunado a esto, las fabáceas son el grupo con el mayor número de especies con importancia económica; numerosas plantas que pertenecen a esta familia son utilizadas cotidianamente en todo el planeta. Entre las más conocidas se puede mencionar al frijol (Phaseolus vulgaris L.), alfalfa (Medicago sativa L.), chícharo (Pisum sativum L.), soya (Glycine max L.), lenteja (Lens culinaris Medik) y jícama (Pachyrhizus erosus (L.) Urb. 1905), entre otras; aunque también la familia cuenta con especies medicinales, de construcción, combustible en forma de leña y ornato (Dorado et al., 2005).

Dentro de los factores que regulan las poblaciones de las fabáceas, los insectos conocidos como brúquidos, gorgojos o escarabajos de las semillas (Coleoptera: Bruchidae), son organismos con biología poco conocida, a excepción de las especies de importancia económica como plagas (Yus-Ramos et al., 2008), cuyas larvas se alimentan y desarrollan en semillas pertenecientes a 34 familias botánicas, con preferencias sobre Fabaceae (Johnson, 1989). En cuanto a la diversidad de especies de la familia Bruchidae a nivel mundial, el grupo se divide en las subfamilias Amblycerinae, Bruchinae, Eubaptinae, Kitorhininae, Pachymerinae y Rhaebinae (Romero \& Johnson, 2004), y actualmente se tienen registradas alrededor de 1,700 especies, distribuidas en 66 géneros, de los cuales, Lugo-García et al. (2015) reportan que, de los 20 géneros y 324 especies presentes en México, 90 especies corresponden al estado de Sinaloa. La importancia económica de los brúquidos radica en su hábito alimenticio espermatófago (Romero, 2002), por lo que juegan un papel importante como reguladores de poblaciones de plantas silvestres al causar daños a más de una especie en las semillas que oscilan entre el 50 y el 100\% (Romero \& Johnson, 2000).

En la última década, se han realizado estudios faunísticos sobre este grupo de insectos para ampliar el conocimiento taxonómico, biológico y ecológico de sus especies, lo cual es clave para determinar su potencial como plagas y su manejo, razón por la cual, la distribución y densidad poblacional relacionada con el cambio del paisaje natural es fundamental para conocer las necesidades de conservación de algunos taxa o la amenaza que podrían representar para las actividades económicas; asimismo, la información sobre la relación entre brúquidos y las semillas de Fabaceae es poco abundante.

Como antecedentes de este tipo de estudios en la región (norte de Sinaloa), está el de Lugo-García et al. (2015), quienes determinaron la diversidad de especies de Bruchidae asociada a semillas de Convolvulaceae y Fabaceae, con registros de 19 especies de bruquidos sobre 29 especies de semillas, distribuidas en los géneros Acanthoscelides (4 especies), Amblycerus (1), Bruchidius (1), Callosobruchus (1), Merobruchus (3), Megacerus (2), Mimosestes (3), Sennius (1), Stator (2) y Zabrotes (1). Los porcentajes de infestación variaron desde 15\% en las Convolvuláceas, Ipomea cholulensis (Kunth) G. Don y Merremia dissecta (Jacq.) Hallier F., y hasta 40\% a las fabáceas Parkinsonia aculeata L., Vigna unguiculata L. y en granos almacenados de garbanzo Cicer arietinum L. se registró a Acanthoscelides desmanthi Johnson, A. guazumae J \& K. y Bruchidius atrolineatus (Pic).

En este sentido, por la importancia que actualmente tienen estas semillas y sus usos potenciales en el futuro, los objetivos del estudio fueron identificar las especies de brúquidos 
López-Mora et al.: Brúquidos asociados a semillas de fabáceas en Sinaloa

asociados a fabáceas y evaluar sus daños sobre la germinación de semillas en cuatro municipios del norte de Sinaloa, México.

\section{MATERIALES Y MÉTODOS}

Se consideró la vegetación natural de fabáceas establecidas en cuatro municipios del norte de Sinaloa: matorral espinoso y dunas costeras de los municipios de Ahome (ubicado entre las coordenadas geográficas $\left.26^{\circ} 02^{\prime} 00^{\prime \prime} \mathrm{N}, 109^{\circ} 01^{\prime} 00^{\prime \prime} \mathrm{O}\right)$ y Guasave ( $25^{\circ} 45^{\prime} 27^{\prime \prime} \mathrm{N}, 108^{\circ} 49^{\prime} 17^{\prime \prime} \mathrm{O}$ ); bosque espinoso en los municipios de El Fuerte ( $26^{\circ} 27^{\prime} 00.5^{\prime \prime} \mathrm{N}, 108^{\circ} 35^{\prime} 20.1^{\prime \prime}$ O) y Choix ( $26^{\circ} 53^{\prime}$ $10.5^{\prime \prime} \mathrm{N}, 108^{\circ} 23^{\prime} 13.8^{\prime \prime} \mathrm{O}$ ). Las localidades consideradas en el estudio tienen altitudes que oscilan entre 0 y $115 \mathrm{~m}$, con pendientes que varían de leves a escarpadas. El clima es de tipo muy seco (BW) y seco (BS), con precipitaciones comprendidas entre 326-607 mm anualmente, con temperaturas promedio que fluctúan entre $23.5-25.6^{\circ} \mathrm{C}$, y extremas mensuales de $4.0-40.7^{\circ} \mathrm{C}$.

Para la identificación de las fabáceas consideradas en el estudio, en enero de 2017 se realizaron recolectas botánicas por triplicado, cortando una rama de la planta con flor y fruto. Las muestras se prensaron y se trasladaron al herbario de la Facultad de Agricultura del Valle del Fuerte (FAVF), de la Universidad Autónoma de Sinaloa (UAS), donde se sometieron a un proceso de secado y preservación para su determinación taxonómica, conforme a claves y criterios de Mc Vaugh (1987), además de ser corroborada por el M. en C. Juan Gaxiola Félix, Coordinador del Herbario de la Facultad de Agricultura del Valle del Fuerte, de la Universidad Autónoma de Sinaloa, con confirmación mediante cotejo de muestras con el Herbario Nacional de la Universidad Nacional Autónoma de México (MEXU).

Para la determinación de especies de brúquidos asociados a fabáceas, durante los meses de febrero a diciembre de 2017, se recolectaron semillas de frutos secos provenientes de fabáceas presentes en el área de estudio. Los frutos recolectados se colocaron en bolsas de papel a las cuales se les separó y etiquetó individualmente con datos como localidad, fecha, especie de fabácea, etc. El material se trasladó y se conservó en el laboratorio de la Colección Entomológica del Valle del Fuerte, de la Universidad Autónoma de Sinaloa (CEVF-UAS), donde una vez depositadas en recipientes de plástico de un litro, se revisaron periódicamente hasta obtener las emergencias de adultos, los cuales se depositaron en tubos eppendorf de $5 \mathrm{ml}$ con alcohol etílico al $70 \%$.

Para la identificación morfológica de los brúquidos se extrajeron las genitalias de los machos, mismas que se procesaron conforme a lo considerado por Kingsolver (1970) y Kingsolver y Whitehead (1974); asimismo, la interpretación de las genitalias se basó en la nomenclatura propuesta por Romero y Johnson (1999); además, se consultaron las colecciones de referencia del Colegio de Postgraduados (CENA), Montecillo, Estado de México y el Instituto de Ciencias de la Benemérita Universidad Autónoma de Puebla. El material identificado fue corroborado por el Dr. Jesús Romero Nápoles, especialista en Bruchidae a nivel internacional. Los ejemplares estudiados se encuentran depositados en las colecciones entomológicas del Colegio de Postgraduados, Montecillo, Estado de México, en la Unidad Académica de Agricultura de la Universidad Autónoma de Nayarit (UAA-UAN) y en la Facultad de Agricultura del Valle del Fuerte (CEVF-UAS). 
Finalmente, para la estimación de daños por especies de brúquidos sobre las semillas de fabáceas, se contabilizó el número total obtenido de los frutos dehiscentes de cada especie de planta en cada uno de los municipios. De estas, se enumeró a aquellas que presentaban el opérculo de emergencia de adultos (brúquidos o parasitoides), así como el número de semillas con orificio de penetración de la larva sin opérculo de emergencia de adultos, con estos datos se obtuvo el porcentaje entre las semillas sanas vs dañadas.

\section{RESULTADOS}

De un total de 68,340 semillas de fabáceas recolectadas pertenecientes a 17 géneros y 25 especies de plantas hospederas, se obtuvieron emergencias de 19,396 brúquidos adultos, los cuales se agruparon en siete géneros y 14 especies (Cuadro 1).

Las afectaciones por estos gorgojos sobre las semillas de Fabáceae en el municipio de Ahome (Sierra de Barobampo) fue de 13 especies de brúquidos asociadas a siete especies vegetales, la especie más perjudicial fue M. santarosae, con $43.9 \%$ y 33.1\% de daños a Mariosouza coultieri y M. acatlensis, respectivamente, seguido por $A$. desmanthi sobre $D$. covillei con daños del $37.07 \%$ (Cuadro 1). En El Fuerte (localidad Ocolome) emergieron siete especies de gorgojos provenientes de semillas de seis especies de fabáceas, de las cuales, las mayormente afectadas fueron S. herbaceae y S. occidentalis, con $38.95 \%$ y $29.48 \%$ de daños causados por $A$. desmanthi, respectivamente, mientras que en $V$. unguiculata, se tuvieron afectaciones del $55.4 \%$ de las semillas por las especies C. maculatus y M. mimosae (Cuadro 1). En Guasave, las siete especies de brúquidos emergieron de seis especies de fabáceas; las mayores afectaciones correspondieron a C. maculatus sobre C. arietinum (41.3\%), M. ulkei sobre P. aculeata (40.6\%) y M. mimosae sobre P. florida (37.7\%) (Cuadro 1). Finalmente, en Choix se registraron siete especies de gorgojos emergidas de semillas pertenecientes a seis especies de fabáceas; las mayores afectaciones correspondieron a $M$. insolitus (34.7\%) sobre A. sinaloensis, seguido por A. acapulcencis (29.1\%) sobre C. cacalaco, y S. pruininus (28.6\%) sobre C. nictitans (Cuadro 1).

Cuadro 1. Relación entre especies de Bruchidae y sus fabáceas hospederas en el norte de Sinaloa, México.

\begin{tabular}{|c|c|c|c|c|}
\hline Municipio & Especie de Bruchidae & $\begin{array}{l}\text { No. de } \\
\text { organismos }\end{array}$ & Planta hospedera & $\begin{array}{l}\text { \% de } \\
\text { daño }\end{array}$ \\
\hline \multirow[t]{7}{*}{ Ahome } & $\begin{array}{l}\text { Merobruchus santarosae } \\
\text { (Kingsolver) }\end{array}$ & 919 & Mariosousa acatlensis (Benth.) & 33.1 \\
\hline & M. santarosae (Kingsolver) & 82 & M. coulteri (Benth.) & 43.9 \\
\hline & $\begin{array}{l}\text { Merobruchus insolitus } \\
\text { (Sharp) }\end{array}$ & 18 & Albizia lebbeck Benth. & 32.1 \\
\hline & Stator limbatus (Horn) & 2 & A. lebbeck Benth. & 32.1 \\
\hline & $\begin{array}{l}\text { Acanthoscelides desmanthi } \\
\text { (Johnson) }\end{array}$ & 325 & $\begin{array}{l}\text { Desmanthus covillei (Britton \& } \\
\text { Rose) }\end{array}$ & 37.0 \\
\hline & A. mankinsi Johnson & 15 & $\begin{array}{l}\text { Leucaena leucocephala (Lam.) de } \\
\text { Wit }\end{array}$ & 15.1 \\
\hline & $\begin{array}{l}\text { A. macrophthalmus } \\
\text { (Schaeffer) }\end{array}$ & 15 & L. leucocephala (Lam.) de Wit & 15.1 \\
\hline
\end{tabular}




\begin{tabular}{|c|c|c|c|c|}
\hline Municipio & Especie de Bruchidae & $\begin{array}{l}\text { No. de } \\
\text { organismos }\end{array}$ & Planta hospedera & $\begin{array}{l}\text { \% de } \\
\text { daño }\end{array}$ \\
\hline & $\begin{array}{l}\text { Mimosestes nubigens } \\
\text { (Motschulsky) }\end{array}$ & 15 & L. leucocephala (Lam.) de Wit & 15.1 \\
\hline & M. insolitus (Sharp) & 492 & Lysiloma divaricata (Jacq.) Macbr. & 37.3 \\
\hline & M. nubigens (Motschulsky) & 13 & L. divaricata (Jacq.) Macbr. & 37.3 \\
\hline & S. limbatus (Horn) & 10 & L. divaricata (Jacq.) Macbr. & 37.3 \\
\hline & Stator pruininus (Horn) & 20 & L. divaricata (Jacq.) Macbr. & 37.3 \\
\hline & $\begin{array}{l}\text { Mimosestes mimosae } \\
\text { (Fabricius) }\end{array}$ & 94 & Mimosa laxiflora S. Wats. & 15.3 \\
\hline & M. insolitus (Sharp) & 15 & M. laxiflora S. Wats. & 15.3 \\
\hline \multirow[t]{8}{*}{ El Fuerte } & M. mimosae (Fabricius) & 891 & $\begin{array}{l}\text { Acacia cochliacantha Humb. \& } \\
\text { Bonpl. ex Willd }\end{array}$ & 27.2 \\
\hline & S. pruininus (Horn) & 5 & $\begin{array}{l}\text { A. cochliacantha Humb. \& Bonpl. } \\
\text { ex Willd }\end{array}$ & 27.2 \\
\hline & M. insolitus (Sharp) & 636 & Phitecellobium sonorae S. Watson & 16.7 \\
\hline & S. limbatus (Horn) & 924 & P. dulce (Roxb.) Benth. & 23.0 \\
\hline & $\begin{array}{l}\text { Callosobruchus maculatus } \\
\text { L. }\end{array}$ & 3,053 & Vigna unguiculata L. & 55.4 \\
\hline & M. mimosae (Fabricius) & 491 & V. unguiculata $\mathrm{L}$. & 55.4 \\
\hline & A. desmanthi (Johnson) & 1,153 & Sesvania herbaceae (P. Mill.) & 38.8 \\
\hline & A. desmanthi (Johnson) & 636 & Senna occidentalis L. & 29.4 \\
\hline \multirow[t]{7}{*}{ Guasave } & M. nubigens (Motschulsky) & 222 & Acacia farnesiana (L.) Willd & 28.1 \\
\hline & M. mimosae (Fabricius) & 2 & A. farnesiana (L.) Willd & 28.1 \\
\hline & C. maculatus L. & 2,651 & Cicer arietinum $\mathrm{L}$. & 41.3 \\
\hline & Zabrotes subfasciatus & 853 & Phaseolus vulgaris L. & 24.7 \\
\hline & M. mimosae (Fabricius) & 741 & $\begin{array}{l}\text { Parkinsonia florida (Benth. ex A. } \\
\text { Grag) }\end{array}$ & 37.3 \\
\hline & Mimosestes ulkei (Horn) & 880 & Parkinsonia aculeata L. & 40.6 \\
\hline & A. desmanthi (Johnson) & 1,182 & Mimosa polystachya L. & 31.5 \\
\hline \multirow[t]{7}{*}{ Choix } & A. desmanthi (Johnson) & 525 & Desmanthus bicornutus S. Watson & 26.6 \\
\hline & Merobruchus boucheri & 116 & $\begin{array}{l}\text { Chloroleucun mangense var. } \\
\text { leucospermum (Brandegee) }\end{array}$ & 7.9 \\
\hline & S. pruininus (Horn) & 38 & $\begin{array}{l}\text { Chamaecrista nictitans (L.) } \\
\text { Moench }\end{array}$ & 28.6 \\
\hline & M. insolitus (Sharp) & 64 & Caesalpinia sclerocarpa Standl. & 23.3 \\
\hline & M. mimosae (Fabricius) & 18 & C. sclerocarpa Standl. & 23.3 \\
\hline & $\begin{array}{l}\text { Amblycerus acapulcensis } \\
\text { (Kingsolver) }\end{array}$ & 488 & C. cacalaco Bonpl. & 29.1 \\
\hline & M. insolitus (Sharp) & 1,197 & Albizia sinaloensis Britton \& Rose & 34.7 \\
\hline
\end{tabular}

\section{DISCUSIÓN}

Los géneros de escarabajos con mayor número de especies fueron Mimosestes, Acanthoscelides y Merobruchus con tres cada uno, seguido por Stator con dos, lo que representa el $78.6 \%$ de las especies; en este sentido, existe cierta relación con lo reportado para los Estados de México y Morelos por Romero et al. (2009), donde Acanthoscelides, Merobruchus, Mimosestes y Stator 
representaron el $53.3 \%$ de las especies emergidas de las semillas, además de obtener la emergencia de cuatro especies de Megacerus, debido posiblemente a que en dicho estudio también se consideraron semillas de especies vegetales comprendidas en las familias Convulvulaceae (Ipomoea triloba) y Sterculiaceae (Guazuma tomentosa).

En cuanto a daños a fabáceas, 17 especies fueron atacadas por una sola especie de brúquido y seis por dos especies; en el caso de L. leucocephala y L. divaricata, éstas son atacadas por cuatro especies. Al respecto, Johnson (1981) registró que el 50\% de Acanthoscelides spp. dañaron solo a una especie de fabácea, mientras que el $60 \%$ de los géneros de plantas hospederas soportan sólo a una especie de Acanthoscelides; a su vez, Janzen (1977), encontró que el 73\% de las especies de brúquidos asociados a fabáceas arborescentes del bosque tropical caducifolio de Costa Rica atacan los granos de una especie, el $15 \%$ de dos y $6 \%$ de tres.

Para el caso particular de la especie $A$. desmanthi en asociación con la fabácea $M$. polystachya en Guasave, los resultados coinciden en cuanto a género con lo reportado por Escobar-Domínguez et al. (2018), quienes mencionan la presencia de A. mexicanus sobre 26 especies de Mimosa en el mundo. Los resultados obtenidos en el El Fuerte coinciden parcialmente con lo obtenido en Irapuato (Guanajuato) por Salas-Araiza et al. (2001), quienes registran para Acacia spp. daños por Stator vachelliae, S. sordidus y Mimosestes nubigens, con daños que oscilaron entre $32 \%$ y $84 \%$, contra el $27.2 \%$ causado a las semillas de $A$. cochliacantha por S. pruiunus y M. mimosae. También se registra coincidencia parcial con lo obtenido en la provincia de Holguín (Cuba) por Sánchez et al. (2017), quienes reportan a las especies Stator bottimeri y Sennius fallax con 32.5\% de infestación sobre Acacia belairioides, contra el 27.2\% de daños obtenidos en el estudio, causados por M. mimosae y S. pruininus sobre A. cochliacantha en el municipio.

En El Fuerte, los resultados coinciden parcialmente con los reportados en Tlaquiltenango, Morelos por Romero-Gómez et al. (2009), al registrar la presencia de M. santarosae y S. limbatus sobre Acacia coulteri A. Grey; por otro lado, los daños causados por S. limbatus sobre $P$. dulce registrados en este estudio en el mismo municipio (23.02\%), difieren notablemente con los obtenidos por los mismos autores en entidades como el Estado de México y Morelos, con daños del $99.3 \%$ por la misma especie de brúquido, debido posiblemente a la ubicación geográfica, altitud y condiciones abióticas imperantes en los años y regiones de estudio, con coincidencia también en el municipio de Guasave en cuanto a presencia de M. nubigens sobre A. farnesiana, reportados en el municipio de Tilzapotla, Morelos.

\section{CONCLUSIONES}

Se identificaron 25 especies de fabáceas: Ahome (7), El Fuerte (6), Guasave (6) y Choix (6). De 68,340 semillas emergieron 19,396 gorgojos adultos, agrupados en siete géneros y 14 especies, con dominancias en especies de Mimosestes (3), Acanthoscelides (3), Merobruchus (3) y Stator (2). De las 25 especies vegetales, 17 de ellas fueron atacadas por una sola especie de brúquido, seis por dos y las restantes dos por una sola especie de Bruchidae. En Ahome, la especie de brúquido más perjudicial fue Merobruchus santarosae, con $43.9 \%$ de daño sobre Mariosouza coulteri y $33.1 \%$ sobre M. acatlensis; en El Fuerte, Acanthoscelides desmanthi causó daños del 38.8\% a Sesvania 
herbaceae y $29.4 \%$ a S. occidentalis; en Guasave, los mayores daños correspondieron a las especies Callosobruchus maculatus (41.3\%) sobre Cicer arietinum, Mimosestes ulkei (40.6\%) sobre Parkinsonia aculeata y Mimosestes mimosae (37.7\%) sobre Parkinsonia florida; en Choix los mayores daños correspondieron a Merobruchus insolitus (34.7\%) sobre Albizia sinaloensis y Amblycerus acapulcencis (29.1\%) sobre Caesalpinia cacalaco.

\section{LITERATURA CITADA}

Aguado, A., Suárez, H. (2006) Impacto del ataque de Algarobius riochama Kingsolver (Coleoptera: Bruchidae) sobre Prosopis juliflora (sw) dc en la zona urbana de Santa Marta. Revista Intrópica, 3, 13-20.

Dorado, O., Arias, D. M., Ramírez, R., Sousa, M. (2005) Leguminosas de la Sierra de Huautla. Universidad Autónoma del Estado de Morelos. CEAMISH, 176 pp.

Escobar-Domínguez, A. A., Romero-Nápoles, J., Equihua-Martínez, A., Carrillo-Sánchez, J. L., Ramírez-Alarcón, S. (2018) Brúquidos (Coleoptera: Bruchidae) asociados a semillas de especies del género Mimosa L. (Leguminosae: Mimosoideae). Acta Zoológica Mexicana (nueva serie), 34, 1-17.

https://doi.org/10.21829/azm.2018.3412149

Harden, G. J. (2002) Fabaceae. Pp. 363-632. En: Harden, G. J. (Ed.). Flora of New South Wales. University of New South Wales, Australia.

Janzen, D. H. (1977) The interaction of seed predators and seed chemistry. Pp. 415-428. En: V. Labeyrie (Ed.). Comportement des insectes et milieu trophique. Publication CNRS (France).

Johnson, C. D. (1981) Interactions between bruchid (Coleoptera) feeding guilds and behavioral patterns of fruit of the Leguminosae. Environmental Entomology, 10, 249-253. https://doi.org/10.1093/ee/10.2.249

Johnson, C. D. (1989) Adaptive radiation of Acanthoscelides in seeds: examples of Legume-Bruchid Interactions. Pp. 747-779. En: C. H. Stirton, J. L. Zarucchi (Eds.). Advances in Legume Biology. Monograph System Botanical. Missouri Botanical Garden.

Kingsolver, J. M. (1970) A study of male genitalia in Bruchidae (Coleoptera). Proceedings of the Biological Society of Washington, 72, 370-386.

Kingsolver, J. M., Whitehead, D. R. (1974) Biosystematics of Central American species of Ctenocolum, a new genus of seed beetles (Coleoptera: Bruchidae). Proceedings of the Biologicals Society of Washington, 87, 283-312.

Lugo-García, G. A., López-Mora, J., Romero-Nápoles, J., Reyes-Olivas, Á., Rodríguez, F., Sánchez-Soto, B. H. (2015) Gorgojos de la familia Bruchidae (Coleóptera) asociados a semillas de cultivos y flora adyacente del norte de Sinaloa, México. Entomología mexicana, 2, 435-441.

Mc Vaugh, R. (1987) Flora Novo-Galiciana: A Descriptive Account of the Vascular Plants of Western Mexico. University of Michigan Press. Vol. 16. 363 pp.

Romero, N. J. (2002) Five new species of Meibomeus Bridwell from the New World with host records for them and six named species (Coleoptera: Bruchidae). The Coleopterist Bulletin, 56 (2), 182-202.

https://doi.org/10.1649/0010-065X(2002)056[0182:FNSOMB]2.0.CO;2 
Romero, N. J., Johnson, C. D. (1999) Zabrotes sylvestris, a new species from the United States and Mexico related to Z. subfasciatus (Boheman) (Coleoptera: Bruchidae: Amblycerinae). The Coleopterist Bulletin, 53 (1), 87-98.

Romero, N. J., Johnson, C. D. (2000) Revision of the genus Zabrotes Horn of Mexico (Coleoptera: Bruchidae: Amblycerinae). Transactions of the American Entomological Society, 126 (2), 221274.

Romero, N. J., Johnson, C. D. (2004) Checklist of the Bruchidae (Insecta: Coleoptera) of Mexico. The Coleopterists Bulletin, 58, 613-635. https://doi.org/10.1649/694

Romero-Gómez, G., Romero-Nápoles, J., Yus-Ramos, R., Burgos-Solorio, A., ValdezCarrasco, J., Flores-Morales, A. (2009) Gorgojos de la familia Bruchidae (Coleoptera) asociados a semillas de plantas silvestres destinadas para germoplasma. Boletín Sociedad Entomológica Aragonesa, 44, 333-342.

Salas-Araiza, M. D., Romero-Nápoles, J., García-Aguilera, E. (2001) Contribución al estudio de los brúquidos (Insecta: Coleoptera) asociados a fabáceas arbustivas. Acta Universitaria, 11 (1), 26-32. https://doi.org/10.15174/au.2001.324

Sánchez, J. A., López, D., Fernández, I., Gómez, J. L., Pernús, M. (2017) Depredación de semillas de Acacia belairioides (Fabaceae) por brúquidos (Coleoptera: Chysomelidae: Bruchinae) y sus efectos en la germinación. Acta Botánica Cubana, 216 (1), 55-61.

Sousa, M., Delgado, A. (1993) Mexican Leguminosae: phytogeography, endemism and origins. Pp. 459-513. En: T. P. Ramamoorthy, R. Bye, J. Fa (Eds.). Biological Diversity of México. Oxford Univ. Press, New York.

Yus-Ramos, R., García-Becerra, R., Ventura-Pérez, D. (2008) Nuevos datos sobre la biología de algunas especies de brúquidos (Coleoptera: Bruchidae) de las Islas Canarias: fitohuéspedes y parasitoides. Boletín Sociedad Entomológica Aragonesa, (42), 355-359. 